\title{
Elemental Morphological and Chemical Characterization of Individual TSP Particles by SEM-EDS
}

\author{
Roberto Ramirez-Leal \\ Sonora State University, Hermosillo, Sonora, Mexico
}

One of the very important air pollutants are particles that are normally suspended in urban atmospheres, can be of natural origin or produced by human activities; these can be introduced into the air as solid or liquid particles or they can be formed by photochemical reactions from contaminants already present.[1]

Traditionally, studies on atmospheric aerosols have focused on their classification based on size, concentration and chemical composition of particle mass; and with relative few details of the dimensions of its size, of the shape and composition of its individual members, which are more directly correlated with its effects on public health and the environment.

So it is very important to know the origin, natural or anthropogenic, of atmospheric particles, because there is currently little information about them; Therefore, the characterization of atmospheric particles based on morphology, size and chemical composition is an appropriate method for studying them.

As each pollutant has characteristics, such as hygroscopicity, water content, refractive index, density and its own physical and chemical properties; So the use of the real morphology of atmospheric particles will allow us greater certainty about the role that particles play in our environment and in plant and animal organisms.[2]

Currently the Scanning Electron Microscope, is a reliable equipment for the study of inorganic particles that are in our atmosphere, and therefore, those that are in contact with the population.

The impact that the use of electrons has had, in the study of particles, has been enormous, since it has allowed us to obtain immense information about their nature, origin and chemical composition.

And because the scanning electron microscope has sufficient specificity for the analysis of small particles and below the range of nanometers; it provides us with the information that has been essential for the observation of aerosols, because we can identify and characterize completely and individually, whatever is of our interest, among a large group of particles.[3]

TSP particles was collected by high-volume sampler, located in the downtown of the city, using a sampling flow of $1.3 \mathrm{~m} 3 / \mathrm{min}$. Sampling was carried for $24-\mathrm{h}$ one day a week. The filters used were fiberglass, with size of $8 \times 10$ inch that captured TSP particles. Analysis of TSP were performed using scanning electron microscopy (SEM) JEOL Model JSM-5800LV coupled with energy dispersive spectrometer (EDS) EDAX DX prime brand, for determination of morphology and elemental composition of airborne particles. Specimens were processed by separating the collected particles from the fiberglass filters by means of submersing a $2 \mathrm{~cm} 2$ section of each filter into isopropilic alcohol within a test tube for 5 minutes.

Then, an aliquot of the suspension was placed over a sample holder, and is introduced into the chamber of SEM.

The aim of the present study is to examine morphology and elemental chemical composition of single TSP particles as part of an air quality study by SEM-EDS. 
the City of Hermosillo is located between latitude $20^{\circ} 01^{\prime} 00^{\prime \prime}$ and $20^{\circ} 08^{\prime} 30^{\prime \prime}$ north latitude and between the meridian $110^{\circ} 54^{\prime} 30^{\prime \prime}$ and $111^{\circ} 01^{\prime} 00^{\prime \prime}$ west longitude at an altitude of $200 \mathrm{~m}$ above sea level; is the state's capital, located to the center-west of the coastal plain, in the northwest region of Mexico.

We obtained 50 images of TSP particles where its morphology and chemical composition of each particle individually was analyzed by SEM-EDS. Figure 1 indicates compositional data of elemental chemical constituents and atomic percentage (At\%) from TSP particles which showed the following elements: Al, $\mathrm{Ba}, \mathrm{Ca}, \mathrm{Ce}, \mathrm{Cl}, \mathrm{Cu}, \mathrm{Fe}, \mathrm{K}, \mathrm{Mg}, \mathrm{Pb}, \mathrm{S}, \mathrm{Si}, \mathrm{Ti}, \mathrm{Zn}$ and $\mathrm{Zr}$; and the predominant elements were $\mathrm{Fe}(29 \mathrm{At} \%)$, Mg (18 At\%), Si (14 At\%) and Al (At13\%), The structure of the particles can be diverse and generally present a two dimensional appearance. (Figure 2). It is often found that such particles have an irregular morphology, with peripheral edges and fracture lines and some with an ovoid shape.

The results of the characterization of the particles obtained from are related to the emission sources located at sampling site, since elements such as $\mathrm{Al}, \mathrm{Si}, \mathrm{Ca}, \mathrm{Fe}, \mathrm{K}$ and $\mathrm{Mg}$ are very abundant in the samples and that indicates an association of lithophilic sources and / or of the earth's crust, as well as agricultural activities, such as areas of soil not affected by human activity, paved streets and areas under construction.

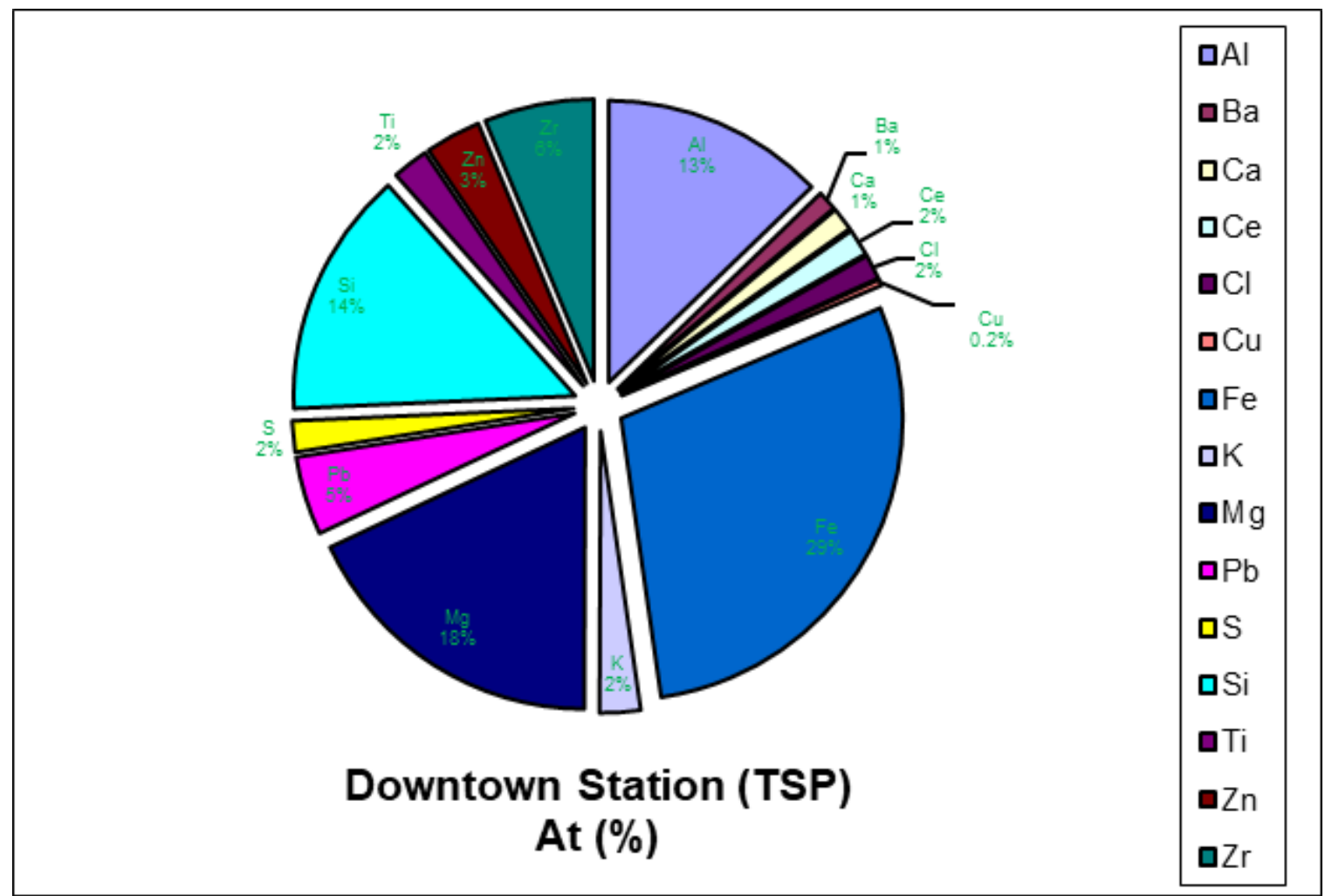

Figure 1. Majority of elemental constituents of TSP Downtown Station samples in urban area (Averaged values of atomic percentage (At\%) for all filter samples) 


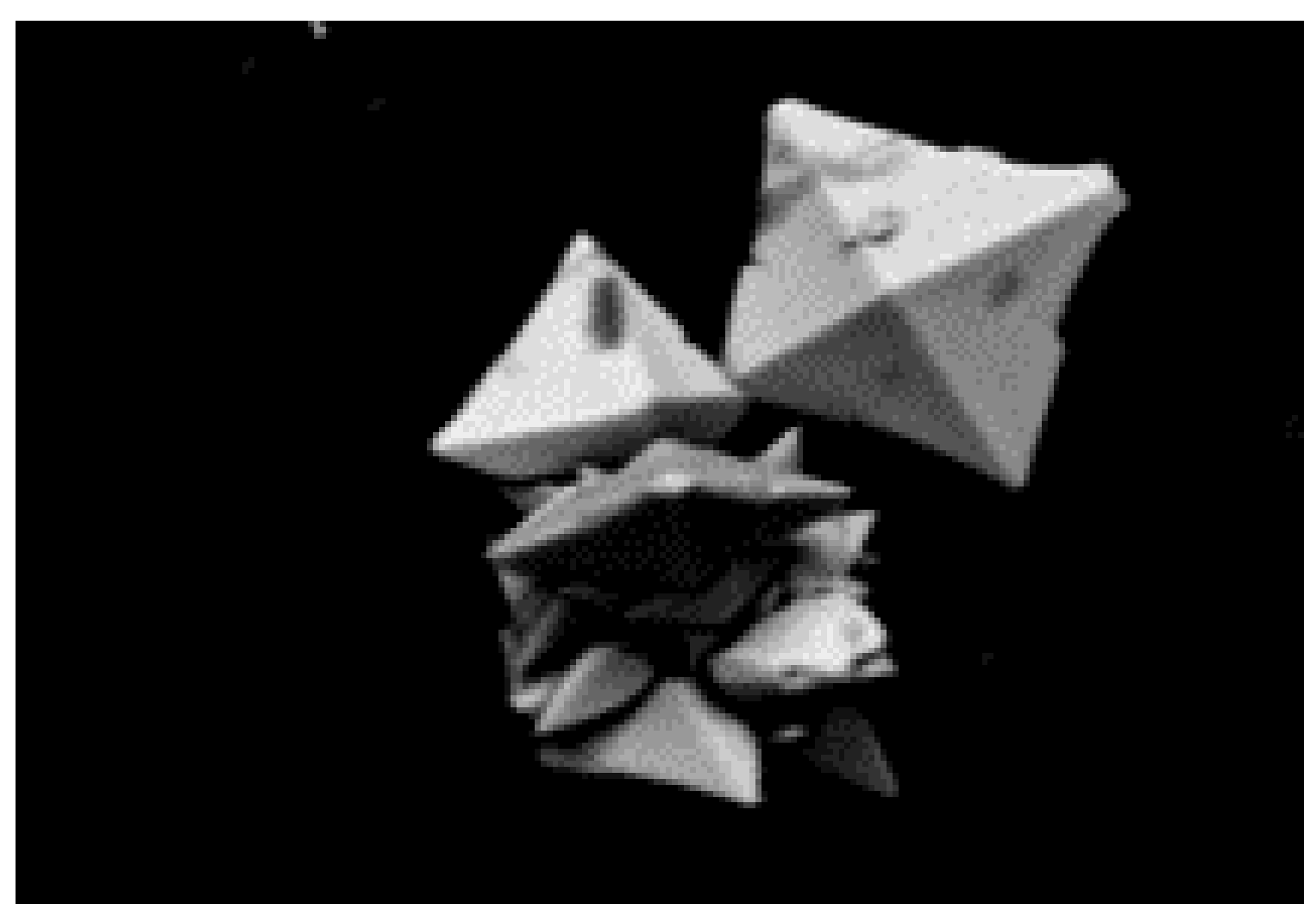

Figure 2. Micrograph of the sample in SEM-EDS. 4000x, BEI and $15 \mathrm{Kv}$

\section{References}

1.- Zhai, Y.B., Fu, Z.M. and Wang, L.F. (2012) Characteristic, Composition, and Sources of TSP Investigated by HRTEM/EDS and ESEM/EDS. Environmental Monitoring and Assessment, 184, 66936707.

2.- Ramirez-Leal, R., Duarte-Tagles, H., Burgos-Hernandezn, M. and Chavez-Toledo, C. (2013) SEMEDS Identification and Characterization of Radioactive Particles in Samples of PM10. Microscopy and Microanalysis, Cambridge University Press, Danvers, 2002-2003,

3.- Ramirez-Leal, R.; Cruz-Campas, M.; Estuardo-Moreno, H.(2018) Characterization of PM10 Particles by SEM-EDS. Microsc. Microanal. 24 (Suppl. 1), 1070. 